\title{
Finite Element Modelling of Central Leader and Vase Shape Cherry Trees
}

\author{
LÁNG, Z. ${ }^{*}$, CSORBA, L. ${ }^{2}$
}

\begin{abstract}
Central leader and vase form tree models were built using Finite Element Modelling (FEM). Their main characteristics were chosen to be the same. To get comparable results to real values, acceleration versus time curves of the two types of real trees were processed using FFT method to determine their natural frequencies. The natural frequencies measured on real trees and calculated for the models have shown good similarity. The models were virtually exposed to the effect of horizontal forced vibration in the frequency range of $0-20 \mathrm{~Hz}$. Acceleration-frequency curves were calculated and drawn to find the best frequency values for the highest accelerations and also to see their differences in the limb. For the same purpose, the direction of shaking was also changed. It was found that for the central leader limb shape multidirectional shaking would bring uniform detachment, while for the vase form trees, even the unidirectional shakers are appropriate. Real trees were also shaken and their acceleration-frequency curves were compared with the values of the FEMs. The resultant good similarity proves the ability of the models. The acceleration values achieved in the vase form models were much higher than for the central leader type. The acceleration-frequency curve of the shaker unit can be used to find the best frequency for shaking.
\end{abstract}

Keywords: fruit tree, modelling, FEM, limb shape, shaker harvesting

\section{Introduction}

Mechanical shaking of a tree is the most widespread harvesting technology for stone fruits. Both limb and trunk shaking are practised. In the first case, more main branches must be shaken. This method is slower, but the removal is more perfect. It also causes less damage to the tree (Fenyvesi and Horváth, 2008).

* Corresponding author. E-mail: zoltan.lang@uni-corvinus.hu

1Professor, Technical Department, Corvinus University of Budapest, Villányi út 3, 1118 Budapest, Hungary;

2Associate Professor, Department of Mechanics, Szent Istvan University, Gödöllő, Hungary 
For shaking the trunk no more than about 10 seconds are needed for clamping and shaking for each to detach most of the fruits.

The shakers are mostly of inertia-type, whereby oscillating masses are attached directly to the trunk of the tree or through a light weigh rod. The oscillation can be achieved basically either by slider-crank mechanism or by counter-rotating masses. When the shaker unit is connected to the tree via the rod, only one direction oscillation can be achieved. Attaching the shaker unit with the counter-rotating masses directly to the trunk makes multidirectional shaking possible. The advantage of the latter is that the whole tree can be shaken in all directions.

In the harvesting practice, large variations are noticed concerning removal at different parts of the limb even at multidirectional shaking. Explanation for this might be, among others, the diversity in geometrical size and structure of the limb. Being a multi-mass dumped swinging system, it has many natural frequencies. Ideally, the shaking should be occurring at different frequencies to get perfect harvesting. To find optimal shaking frequencies, Yung and Fridley (1975) created a finite element tree model. It consisted of a trunk and two branches, each of them supporting fruit. Natural frequencies, mode shapes, dynamic internal stress and vibration response of the complete tree structure were evaluated for that part of tree at steady-state forced vibration. Liang et al. (1971) Hussain et al. (1975) found that the tree must be shaken at several frequencies to develop vibrations throughout the tree.

The aim of the research described in this paper was to compare two commonly grown cherry tree shapes: the central leader type (Hrotkó et al. 1999) and the vase form, from the point of view of optimal shaking frequencies and to help the decision between limb shapes and shaker harvester. For this purpose - different to the models of Yung and Fridley, Liang et al., Hussain et al. and Phillips et al. (1970) - we have built two whole roottrunk-limb finite element dynamic models to enable the study of natural frequencies of the main structural elements of the model trees and the effect of a steady-state forced vibration in two horizontal directions. Field experiments were planned to be able to compare a model and real tree data.

\section{Materials and Methods}

Finite element model of a central leader and a vase type cherry tree was built of trunk, straight primary and secondary limbs, as well as straight primary and secondary roots. Figure 1 explains the construction of the mod- 
els on the example of the vase form tree. The simplified structure of the two types of trees is shown on Figs 2 and 3.

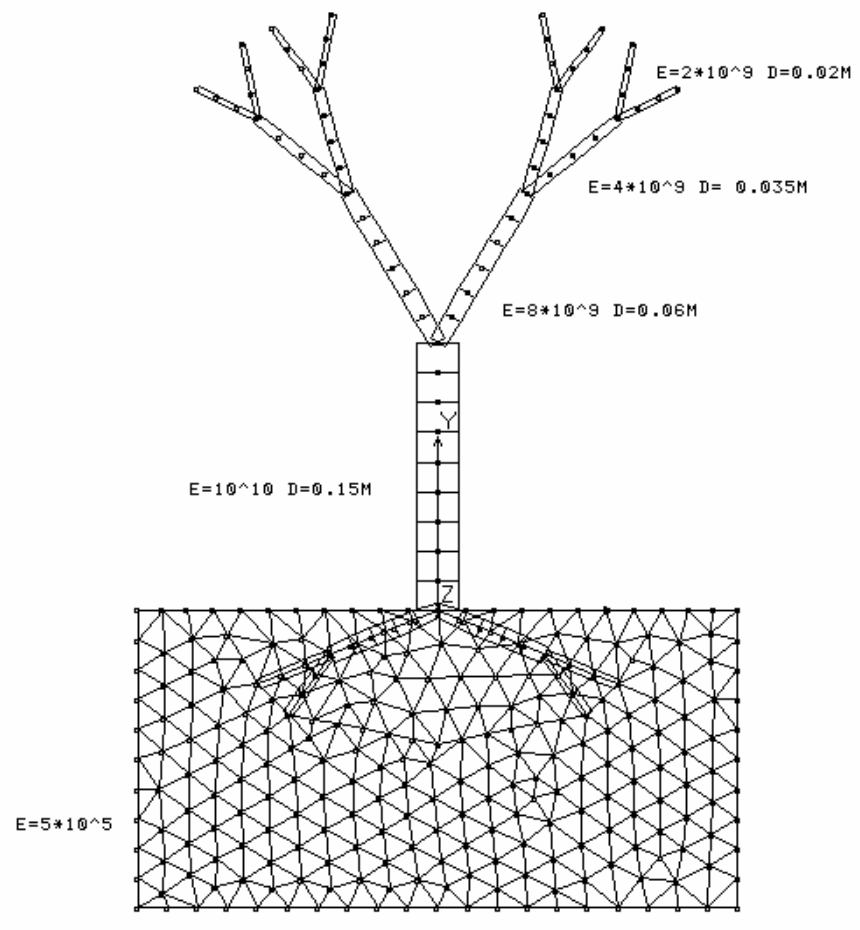

Fig. 1. The construction of finite element models

To be able to compare the two structures most of the characteristics were chosen to be equal. Both were symmetrical around their central axis. Both structures consisted of app. $10 \mathrm{~cm}$ long cylindrical elements. The diameter of the cylinders changed as shown on Fig. 1. This way at any height the diameter of the elements in the two models was the same. Their total height was $2.21 \mathrm{~m}$, their total mass $102.5 \mathrm{~kg}$ at a density of $1000 \mathrm{kgm}^{-3}$. Young's modulus was uniform for the two models but it decreased with the height (see Fig. 1, values in Pa). The Poisson's constant was set to 3.57 for each element, which is an average value for fruit trees. Considering earlier field experiment results (Láng, 2003), linear dumping was chosen throughout the model; the value of Lehr's dumping was set to 0.2. Both models were connected to the soil via four main roots with two secondary roots at the end of each. The secondary roots were fixed to the soil by their surfaces. The main roots were regarded as prolongations of the trunk, so their E values were chosen the same as for the trunk. 
The central leader model consisted of a central trunk and of three main branches in two levels symmetrically distributed. Their position to the horizon was $30^{\circ}$. The branches C4 and C6 stand in the $x y$ plain. At half length the main branches were transformed into two secondary branches and then at half-length it bifurcated again.

The vase type model was constructed of a trunk and four main branches, two of them in plane $x y$, two in plane $y z$. The tilted parts stand in $60^{\circ}$ to the horizon. The formation of secondary and further branches was the same as described for the central leader model.

The trunks of the two finite element models were first virtually exposed to the effect of horizontal forced vibration in two different directions, of the following formula:

$$
F_{f}=F_{0} \cdot \sin (2 \cdot \pi \cdot f \cdot t)
$$

where $F_{0}=3000 \mathrm{~N}$ was the maximal force, acting at $80 \mathrm{~cm}$ above ground.

$0<f<20 \mathrm{~Hz}$ was the chosen frequency range.

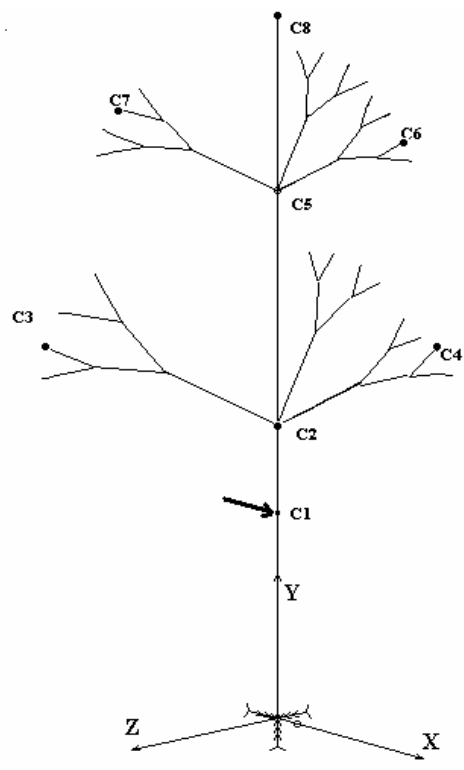

Fig. 2. Model of a central leader type cherry tree

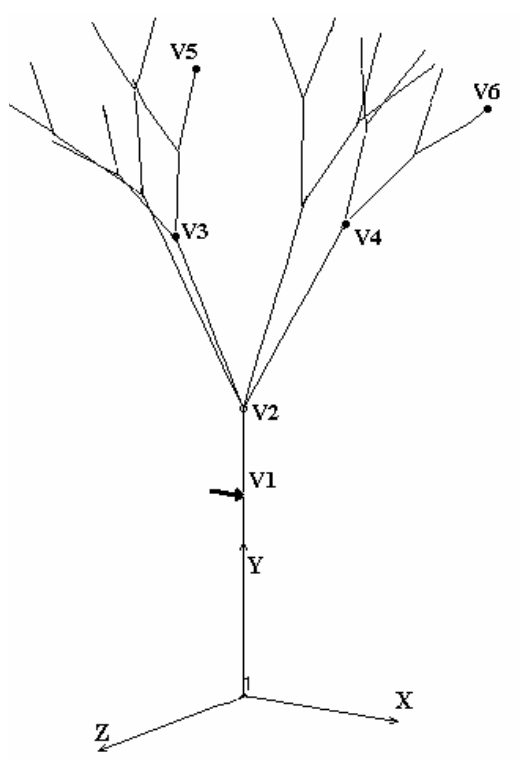

Fig. 3. Model of a vase type cherry tree

In the case of central leader type model, the virtual excitation was first carried out in direction $x$, then in $30^{\circ}$ to it. This way we had branches shaken in their own plane, perpendicular and in $30^{\circ}$ to it. 
The vase form model was first exposed to forced vibration in direction $x$ then in $45^{\circ}$ direction to $x$. This way again we had branches shaken in their own plane, perpendicular and in $45^{\circ}$ to it.
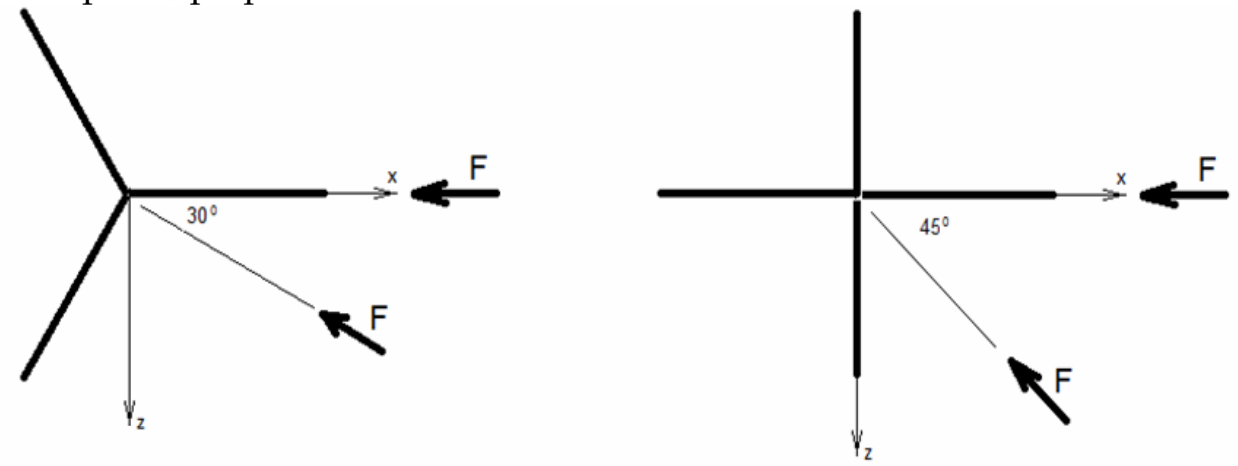

Fig. 4. The directions of virtual shaking of central leader type (left) and of vase form model

To be able to compare the model with real tree, the above procedure was repeated with a generated force, as the inertia type shaker act on the tree:

$$
F_{s}=m \cdot r \cdot(2 \cdot \pi \cdot f)^{2} \cdot \cos (2 \cdot \pi \cdot f \cdot t)
$$

where $m$ and $r$ are the mass and eccentricity of a virtual inertia type shaker.

$0<f<20 \mathrm{~Hz}$ was the chosen frequency range.

Extra masses were added virtually to the clamping points of the models to see their effect onto the optimal shaking frequencies. This may give an idea about the effect of shaker machine mass on the natural frequencies of the machine-tree system.

To get comparable results from real values, field experiments were carried out on 8-year-old central leader and 9-year-old vase type cherry trees after harvest (no fruit on the trees). The geometrical sizes of the samples were similar to those of the models: the selected central leader type trees had an average trunk diameter of $14.7 \mathrm{~cm}$, the vase form trees $15.2 \mathrm{~cm}$; in both cases the number of samples was 10 .

To find the natural frequencies of the trees, their free swing was studied. Accelerometers were fixed on the trees, on places identical to the nodes 1, 4 and 6 of the models (see Figs 2 and 3). The stems were quickly prestressed at $80 \mathrm{~cm}$ above ground by a force of approximately $3000 \mathrm{~N}$ and released. The acceleration versus time run out curves were processed using FFT method, to determine the natural frequencies of the examined parts. 
The acceleration of trunk, primary and secondary limb at different shaking frequencies was recorded in field experiments on vase form trees using inertia type shaker. For this purpose, accelerometers were left on the same places on the tree as said before. Shaking was carried out at different frequencies and the maximal accelerations versus frequency data were plotted for those nodes.

\section{Results and Discussion}

The FEM program made possible the calculation of the system's natural frequencies for both examined limb types. Similar virtual and real values for the central leader type tree are shown in Table 1. According to mechanical harvesting practice, the examined frequency range was chosen at $0-20 \mathrm{~Hz}$.

Table 1. Natural frequencies for the Central leader type tree obtained from FEM and from field test

\begin{tabular}{|c|c|}
\hline \multicolumn{2}{|c|}{ Natural frequencies $(\mathrm{Hz})$} \\
\hline FEM & Field test result \\
\hline 1.70 & 1.56 \\
\hline 3.98 & 4.68 \\
\hline 5.32 & 5.47 \\
\hline 6.54 & \\
\hline 7.27 & 7.81 \\
\hline 7.28 & \\
\hline 10.03 & 10.15 \\
\hline 10.08 & 10.93 \\
\hline 11.50 & 14.06 \\
\hline 13.48 & 14.84 \\
\hline & 15.62 \\
\hline & 18.75 \\
\hline
\end{tabular}

Similar FEM calculated and field test natural frequencies for the vase form structure are shown in Table 2. The examined range here again was $0-20 \mathrm{~Hz}$. 
Table 2. Natural frequencies for the vase type tree obtained from FEM and from field test

\begin{tabular}{|c|c|}
\hline \multicolumn{2}{|c|}{ Natural frequencies $(\mathrm{Hz})$} \\
\hline FEM & Field test result \\
\hline 1.61 & 1.56 \\
\hline 4.05 & 3.91 \\
\hline 5.76 & \\
\hline 6.89 & \\
\hline 8.26 & 8.59 \\
\hline & 10.15 \\
\hline 12.19 & 10.94 \\
\hline 14.04 & 13.30 \\
\hline & 15.62 \\
\hline & 17.18 \\
\hline & 17.68 \\
\hline
\end{tabular}

Figures 5 to 8 show the acceleration-frequency diagrams for both models and for all mentioned directions of shaking, when the shaking force according to Eqn. 1 was virtually applied. The curves were drawn by the FEM program. Acceleration means in these cases their maximal values, in other words, their amplitudes.

Figure 5 shows the acceleration vs. frequency curves of the most important nodes on Central leader type tree model. The direction of virtual shaking was here parallel to the $x$-axes.

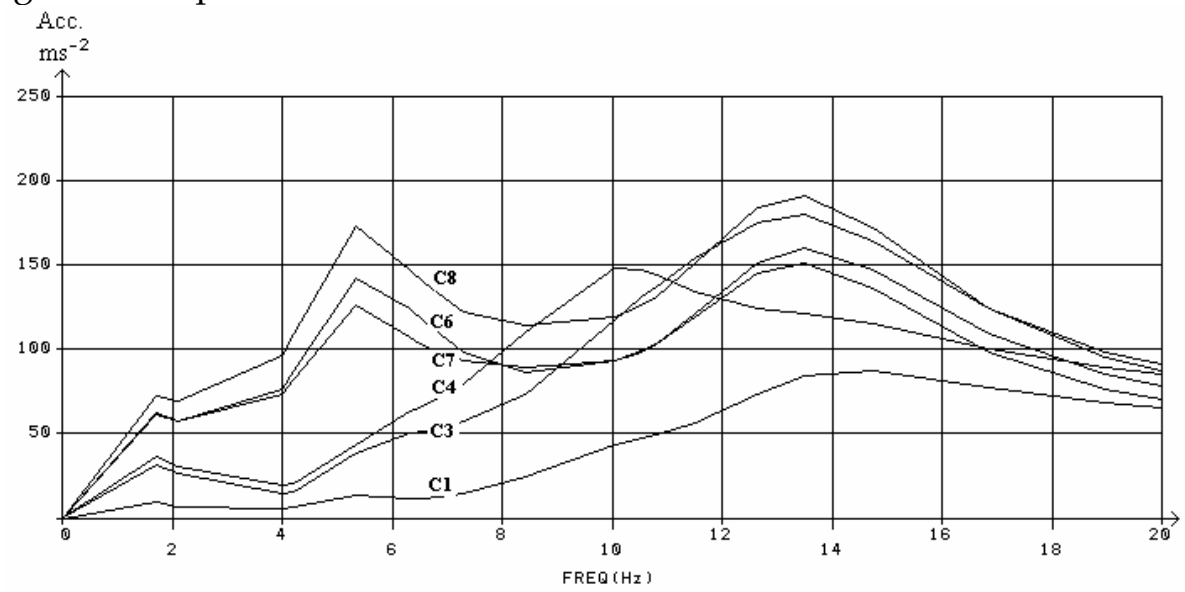

Fig. 5. Acceleration-frequency curves of the nodes of central leader type model. Direction of steady-state forced vibration: $x$. C6 runs throughout higher than C7 
There are two maximums on most of the curves: at about $5.3 \mathrm{~Hz}$ and at about $13.5 \mathrm{~Hz}$. The highest acceleration values were calculated for the top of the limb (C8) and for $\mathrm{C} 3$, which is at the end of the branch, standing in $60^{\circ}$ to the direction of shaking. For the branch in the direction of shaking, the frequency of maximal acceleration is at about $10 \mathrm{~Hz}$ (C4).

Figure 6 presents the acceleration curves of the same model for the case when the direction of forced vibration is $30^{\circ}$ to the axis $x$. Now the highest acceleration appeared at the end of the branch perpendicular to the direction of shaking (C3). The shape of the acceleration/frequency curve of the branch C4 has changed: its maximum keeps from 10 to $13.5 \mathrm{~Hz}$. The two acceleration peaks are at the same frequency values as in Fig. 5.

Figures 5 and 6 include all possible maximal acceleration values of the nodes in the range $0-20 \mathrm{~Hz}$.

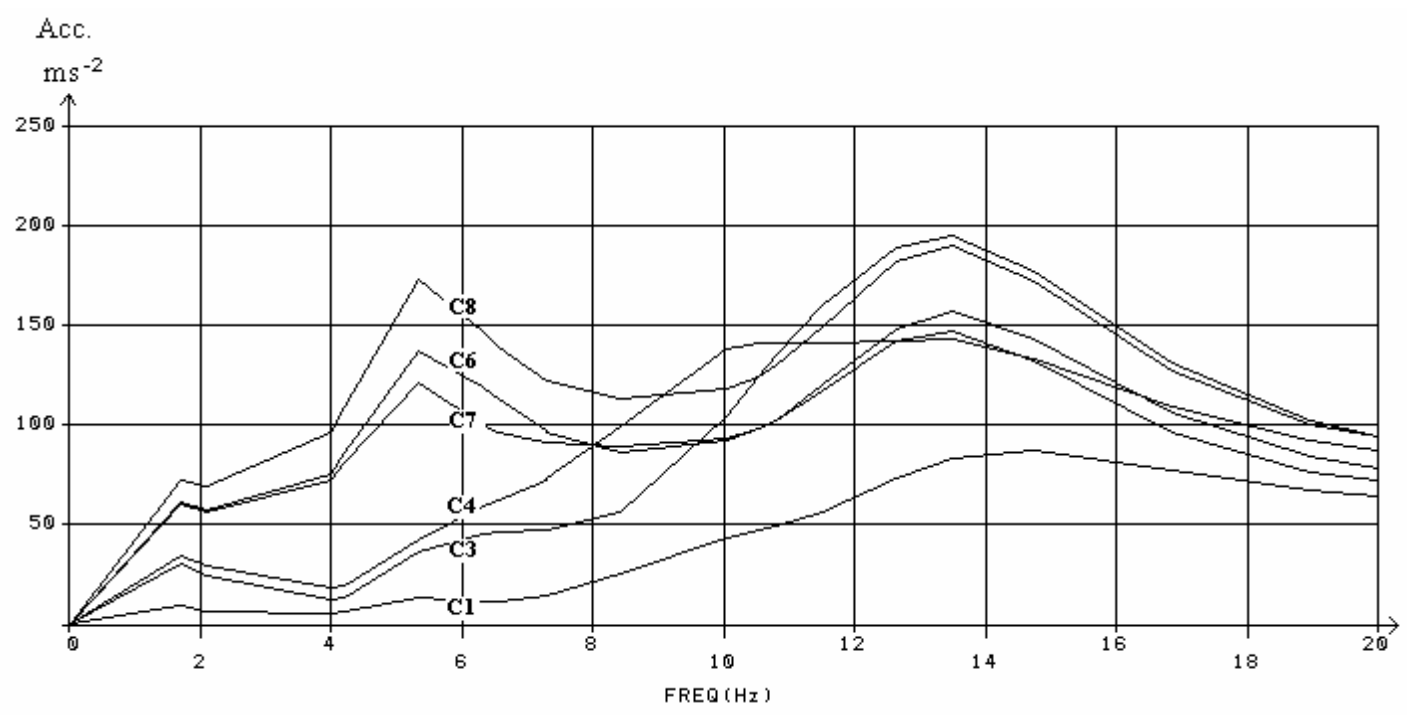

Fig. 6. Acceleration-frequency curves of the nodes of central leader type model. Direction of steady-state forced vibration is $30^{\circ}$ to $x$

Table 3 shows their values at the frequencies 5.3 and $13.5 \mathrm{~Hz}$, for both direction of excitation. 
Table 3. Maximal node acceleration values in $\mathrm{ms}^{-2}$ at 5.3 and $13.5 \mathrm{~Hz}$ excitation in direction $x$ and $30^{\circ}$ to $x$. Model type: Central leader

\begin{tabular}{|c|c|c|c|c|}
\hline \multirow{2}{*}{ Nodes } & \multicolumn{2}{|c|}{ Excitation in direction $x$} & \multicolumn{2}{c|}{ Excitation in direction $30^{\circ}$ to $x$} \\
\cline { 2 - 5 } & $5.3 \mathrm{~Hz}$ & $13.5 \mathrm{~Hz}$ & $5.3 \mathrm{~Hz}$ & $13.5 \mathrm{~Hz}$ \\
\hline C3 & 35 & 170 & 35 & 185 \\
\hline C4 & 45 & 120 & 40 & 135 \\
\hline C6 & 125 & 160 & 135 & 160 \\
\hline C7 & 140 & 150 & 120 & 145 \\
\hline C8 & 170 & 180 & 170 & 180 \\
\hline
\end{tabular}

Because of symmetry the accelerations at the peak (C8) are the same in both cases. At $13.5 \mathrm{~Hz}$ frequency there is not much difference between $\mathrm{C} 7$ and C6. The difference between the accelerations of C3 and C4 is much higher: it makes almost $30 \%$. That means that in order to achieve uniform detachment around the tree, multidirectional shaking has to be applied.

The optimal shaking frequency can be found even during shaking the tree. One has to increase it continuously and, at the same time, monitor the acceleration of the clamp of the shaker (C1). The optimum is where the curve starts to decline (at about $15 \mathrm{~Hz}$ ).

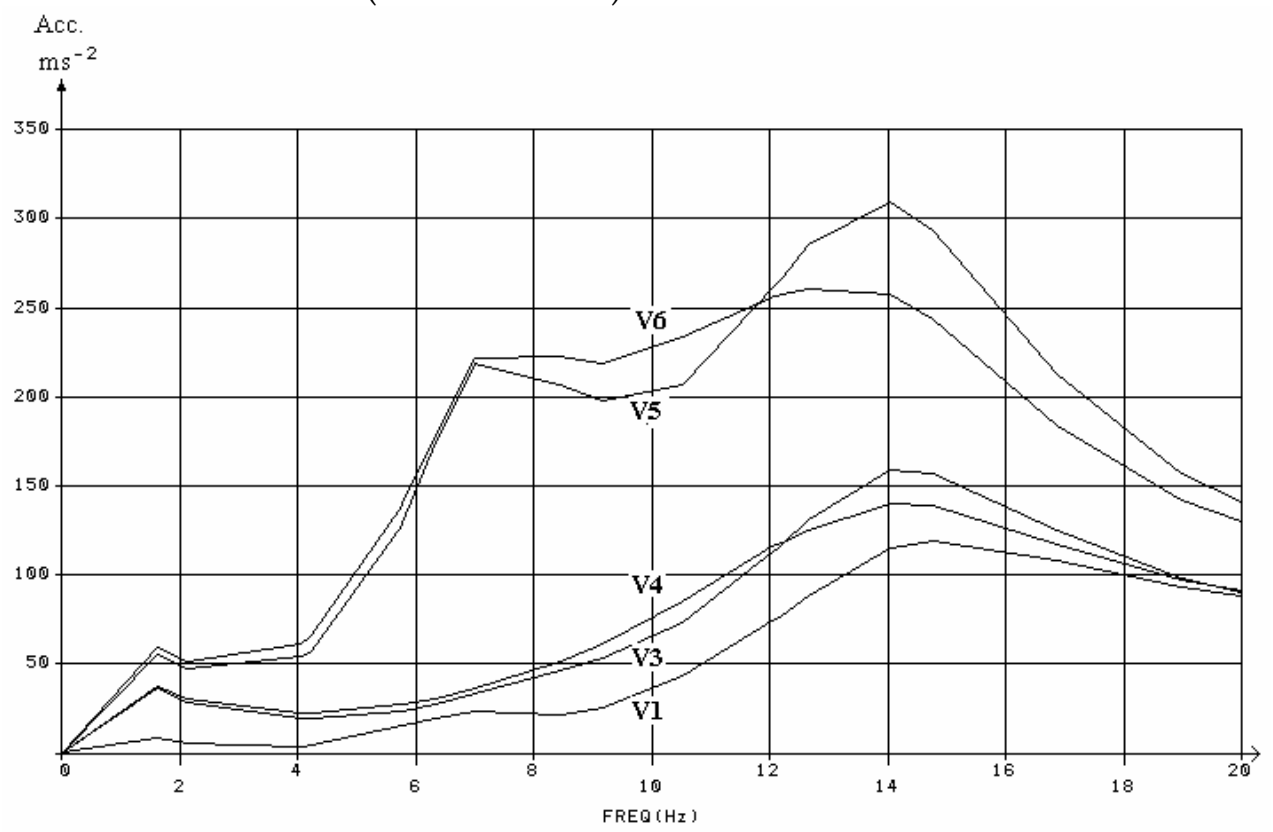

Fig. 7. Acceleration versus frequency curves for the vase type model.

Direction of shaking: $x$ 
The maximal acceleration versus frequency curves for the vase type model are shown in Figs. 7 and 8. Figure 7 shows the case when the direction of shaking is $x$. The two frequency peaks belong to the tips of the main branches V6 and V5 in the plane $x y$ and $y z$. The nodes V3 and V4 reach their maximum acceleration at the second frequency value.

Shaking in direction $z$ the curve of V6 would change place with the curve V5, similarly V4 with V3 (not shown).

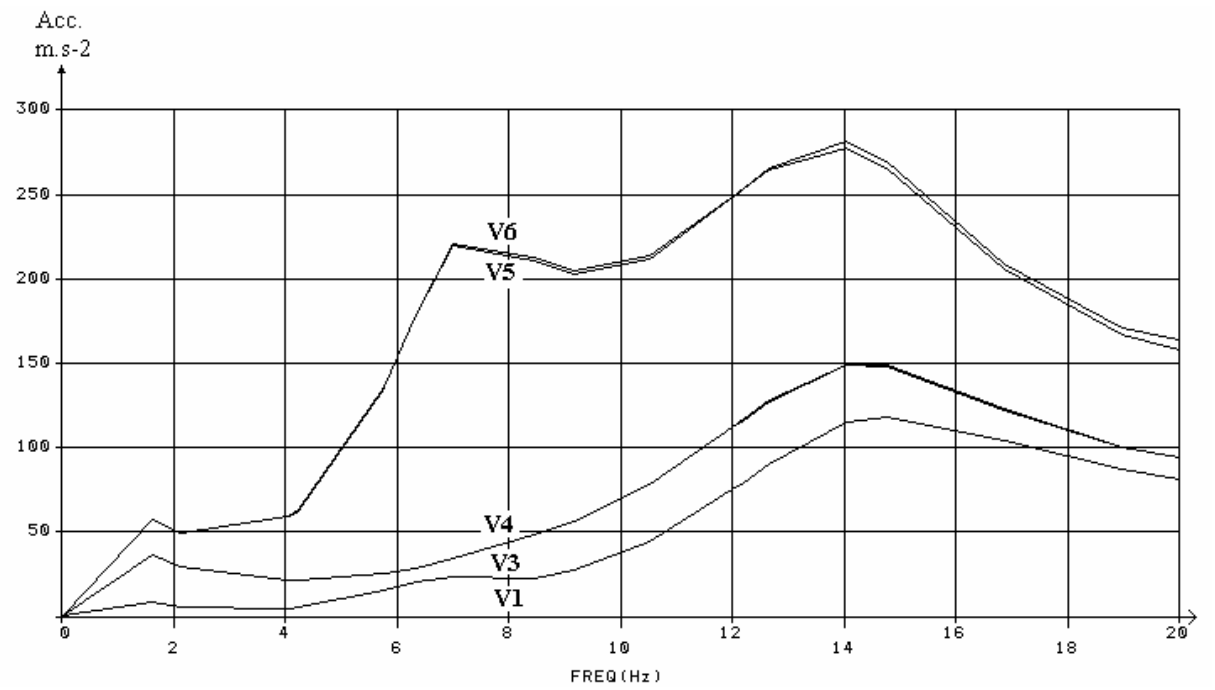

Fig. 8. Acceleration versus frequency curves for the vase type model. Direction of shaking: $45^{\circ}$ to $x$

Some change can be noticed when the direction of shaking is turned $45^{\circ}$ to $x$. Figure 8 shows that case. Although the peaks of curves for V5 (the same as V6) and V4 (the same as V3) are approximately at the same frequencies, their acceleration values are different.

In Figs 7 and 8 all possible acceleration varieties for the nodes in the range of $0-20 \mathrm{~Hz}$ are presented. Table 4 shows their maximum values. It looks clear that when shaking the trees in direction $45^{\circ}$ to $x$ axes, all the 4 limbs are shaken uniformly. In the worst case, (shaking in direction $x$ ) the difference in acceleration of V3 and V4 is less than 13\%. Consequently, unidirectional shakers can harvest the vase form trees effectively. The optimal frequency for shaking is here again at the declination of the curve V1 above the frequency about $15 \mathrm{~Hz}$. In order to find it, the shaking frequency has to be increased constantly and monitored on the shaker device. 
Finite Element Modelling of Central Leader and Vase Shape Cherry Trees

Table 4. Node acceleration values in $\mathrm{ms}^{-2}$ at 7.0 and $14.0-14.5 \mathrm{~Hz}$ excitation in direction $x$ and $45^{\circ}$. Model type: vase

\begin{tabular}{|c|c|c|c|c|}
\hline \multirow{2}{*}{ Nodes } & \multicolumn{2}{|c|}{ Excitation in direction $x$} & \multicolumn{2}{c|}{ Excitation in direction $45^{\circ}$ to $x$} \\
\cline { 2 - 5 } & $7.0 \mathrm{~Hz}$ & $13.8-14.5 \mathrm{~Hz}$ & $7.0 \mathrm{~Hz}$ & $13.8-14.5 \mathrm{~Hz}$ \\
\hline V3 & 30 & 160 & 35 & 150 \\
\hline V4 & 35 & 140 & 35 & 150 \\
\hline V5 & 215 & 310 & 220 & 280 \\
\hline V6 & 220 & 210 & 220 & 280 \\
\hline
\end{tabular}
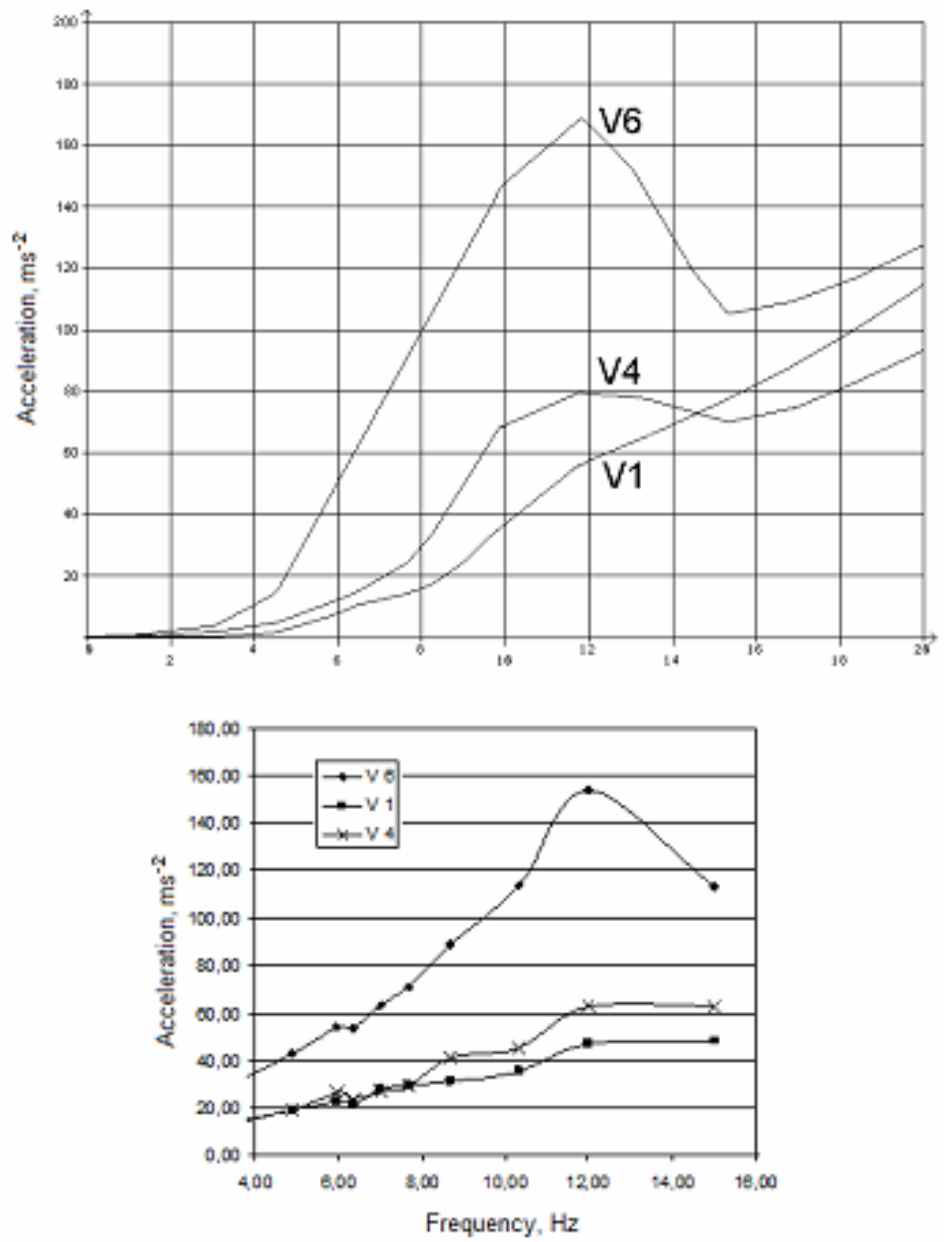

Fig. 9. Acceleration versus frequency curves for the vase type model, shaken virtually (in the top) and actually by an inertia shaker in direction $x$ 
Using a virtual inertia shaker with rotating masses (Equation 2), the acceleration versus frequency curves change significantly. In the examined frequency range, only one peak appears clearly. Due to the second power of frequency in Equation 2, the change in acceleration is rapid. In the top of Fig. 9, typical acceleration/frequency curves are shown for the vase type model drawn by the FEM. In the bottom acceleration versus frequency curves drawn off the field measurement data are presented. Although the curves for the tree model and real cherry tree are not totally the same, their tendency is similar. The dissimilarity may be explained by the differences in geometrical sizes, shapes, structures, total masses and mass-distributions.

By attaching virtually different size of extra masses to the clamping point $(\mathrm{C} 1, \mathrm{~V} 1)$ of the models and drawing the calculated acceleration maximums versus changing mass values, the curves presented in Fig. 10 can be obtained.

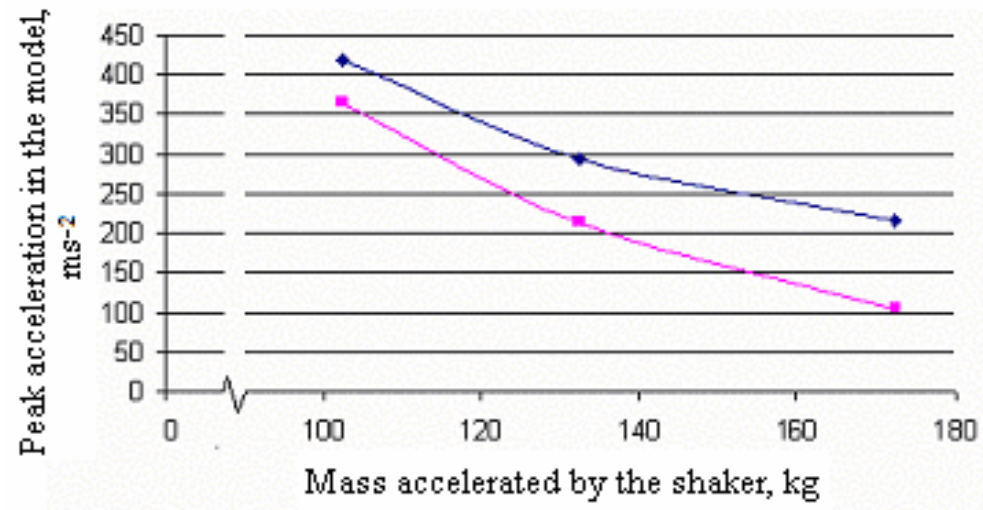

Fig. 10. Peak acceleration versus accelerated mass curves for the vase form (top) and central leader model (bottom)

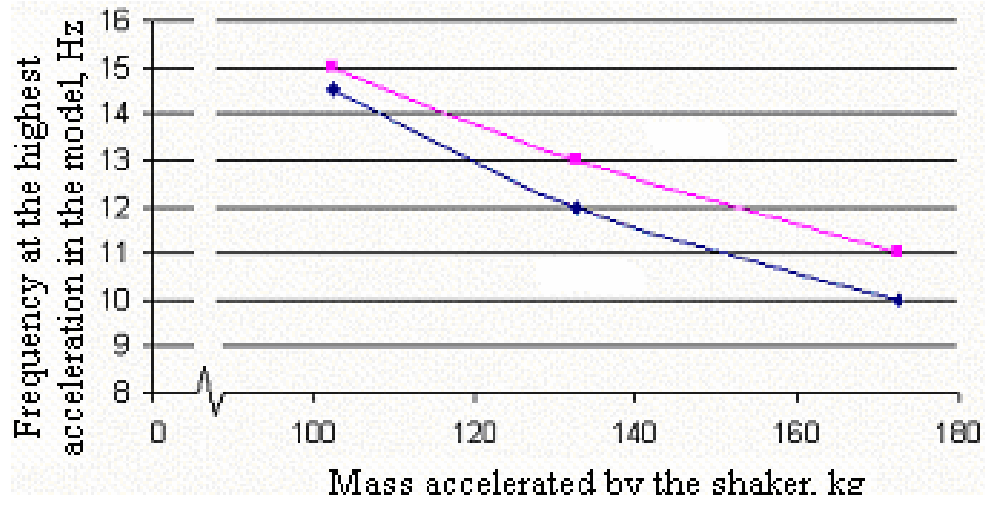

Fig. 11. Frequency at peak acceleration versus accelerated mass curves for the central leader (top) and vase form model (bottom) 
The size of accelerated mass influences the frequency belonging to the peak value as well. Figure 11 shows their relation.

\section{Conclusion}

The comparison of natural frequencies measured on real trees and calculated for similar size finite element models, composed of roots, trunk, primary and secondary limbs, have shown good similarity. It indicates the model's applicability for the replacement of the trees in vibration studies.

Shaking virtually the FEM models in the frequency range of $0-20 \mathrm{~Hz}$ made it possible to calculate and plot the acceleration amplitude response of their most characteristic nods. At the maximums of the frequencyacceleration curves, the detachment of the fruit would be the most likely: those can be regarded as optimal shaking frequencies.

Virtual multi-directional shaking of the models has shown how the direction of excitation influences the acceleration values of the examined nodes.

For the central leader limb, shape differences of $30 \%$ were found. It means the direction of shaking should change during harvest to achieve uniform acceleration throughout the limb. For this kind of a tree, shape multi-directional shakers are recommended.

For the vase form limb, the differences were much lower. In this case, the unidirectional shakers are appropriate.

Comparing the calculated acceleration peaks for the two FEM trees: at the same steady-state horizontal forced vibration, much higher values were found for the vase form. It suggests that the fruits from the vase form trees are easier to detach.

The frequency-acceleration curves also give indication on the optimal shaking frequency. It coincides with the frequency where the shaker unit's acceleration starts to decline.

Frequency-acceleration curves for the tree model and for real cherry tree are running similarly, which proves the suitability of the FEM models.

The FEM models can be used also to study the effect of shaker machine mass on the magnitude of acceleration at the optimal shaking frequency and also the effect of machine mass on the optimal shaking frequency. 


\section{References}

Fenyvesi L, Horváth E. (2008): Basics of mechanical fruit harvesting. In: Sitkei Gy: Technical aspects of horticultural production. Gödöllő: MGI BOOKS, pp. 23-107.

Hrotkó K., Magyar L. and Simon G. (1999): Growth and yield of sweet cherry trees of different rootstocks. Int. Journal of Hortic. Science (3-4): 98-104

Hussain, A.A.M., Rehkugler, G.E. and Gunkel, W.W. (1975): Tree limb response to a periodic discontinuous sinusoidal displacement. Trans. ASAE 18(4), 614-617.

Láng Z. (2003): A fruit tree stability model for static and dynamic loading. Byosystem Engineering 85(4), 461-465.

Liang, T., Lewis, D.K., Wang, J.K. and Monroe G.E. (1971): Random function modeling of macadamia nut removal by multiple frequency vibration. Trans. ASAE 14(6), 1175-1179.

Phillips, A.L., Hutchinson, J.R. and Fridley, R.B. (1970): Formulation of forced vibrations of tree limbs with secondary branches. Trans. ASAE 13(1), 138-142.

Young, C. and Fridley, R.B. (1975): Simulation of vibration of whole tree systems using finite elements. Trans. ASAE 8(3), 475-481. 